\title{
Use of the waist-to-height-ratio to predict cardiovascular risk in patients with diabetes: results from ADVANCE-ON
}

Karin Rådholm ${ }^{1,2}$, John Chalmers ${ }^{2}$, Toshiaki Ohkuma ${ }^{2,3}$, Sanne Peters ${ }^{4}$, Neil Poulter ${ }^{5}$, Pavel Hamet $^{6}$, Stephen Harrap ${ }^{7}$, Mark Woodward ${ }^{2,4,9}$

${ }^{1}$ Division of Community Medicine, Primary Care, Department of Medicine and Health Sciences, Faculty of Health Sciences, Linköping University, Department of Local Care West, County Council of Östergötland, Linköping, Sweden

${ }^{2}$ The George Institute for Global Health, University of New South Wales, Sydney, Australia

${ }^{3}$ Department of Medicine and Clinical Science, Graduate School of Medical Sciences, Kyushu University, Fukuoka, Japan.

${ }^{4}$ The George Institute for Global Health, University of Oxford, UK

${ }^{5}$ International Centre for Circulatory Health, Imperial College, London, U.K.

${ }^{6}$ Montreal Diabetes Research Centre, Centre Hospitalier de l'Université de Montréal, Montreal, Québec, Canada

${ }^{7}$ The University of Melbourne and Royal Melbourne Hospital, Melbourne, Victoria, Australia

${ }^{9}$ Department of Epidemiology, Johns Hopkins University, Baltimore MD, USA

Abstract word count: 248

Word count: 2266

\section{Corresponding author}

Karin Rådholm

Linköping University

SE-581 83 Linköping

This is the author manuscript accepted for publication and has undergone full peer review but has not been through the copyediting, typesetting, pagination and proofreading process, which may lead to differences between this version and the Version of Record. Please cite this article as doi: $10.1111 /$ dom.13311

This article is protected by copyright. All rights reserved. 


\section{Sweden}

Phone: +46101030000

Fax: +46101034020

E-mail: karin.radholm@liu.se; kradholm@georgeinstitute.org.au

This article is protected by copyright. All rights reserved. 


\section{Abstract}

\section{Background}

Patients with type 2 diabetes have a high risk of cardiovascular disease (CVD). Central obesity has been particularly associated with this risk relationship. We aimed to evaluate waist to height ratio (WHtR) as a predictor of risk in such patients.

\section{Methods}

WHtR was evaluated as a predictor for the risk of CVD and mortality amongst 11125 participants with type 2 diabetes in the ADVANCE and ADVANCE-ON studies, and compared with body mass index (BMI), waist circumference and waist hip ratio (WHR). The primary outcome was a composite of death from CVD, nonfatal myocardial infarction or nonfatal stroke. Secondary outcomes were myocardial infarction, stroke, cardiovascular death and death from any cause. Cox models were used, with bootstrapping to compare associations between anthropometric measures for the primary outcome.

\section{Results}

Median follow-up time was 9.0 years. There was a positive association between WHtR and adverse outcomes. The hazard ratio (HR) (confidence interval), per SD higher WHtR, was $1.16(1.11-1.22)$ for the primary endpoint, with no heterogeneity by sex or region, but a stronger effect in people aged 66 years or older. The other three anthropometric measurements showed similar associations, although there was evidence that WHtR marginally outperformed BMI and WHR. Based on commonly used BMI cut points the equivalent WHtR cut points were estimated to be 0.55 and 0.6 , with no evidence of a difference across subgroups. 


\section{Conclusions}

In patients with diabetes, WHtR is a useful indicator of future adverse risk, with similar effects in different population subgroups.

This article is protected by copyright. All rights reserved. 


\section{Introduction}

Overweight and obesity are strongly associated with cardiovascular disease and type 2 diabetes (1-5). Several different anthropometrical measurements are used in the clinical setting to predict disease risk in relation to overweight and obesity. Body mass index (BMI) is well known and frequently used both in clinical practice and in the health and fitness industry. However, BMI is a measure of general adiposity which does not distinguish between visceral and central obesity. Although use of the same risk BMI cut points are recommended for all populations, different cut offs depending on ethnicity have also been suggested (1, 6-9). Waist circumference (WC) does measure central obesity, but the cut offs suggested for its clinical evaluation differ by sex and region (7). Waist to height ratio (WHtR) was suggested in the mid-1990s as an alternative anthropometric measurement (10) that may improve on WC because it takes body size into account $(10,11)$. It has been suggested that WHtR values of 0.5 or above may be used consistently to indicate increased cardiovascular disease risk across sub-populations defined by ethnicity, age and sex (12-15), as well as in children and adolescents $(11,16)$. An additional cut-point value of 0.6 has previously been suggested to predict substantial disease risk $(10,17)$, but this has not gained widespread use.

Type 2 diabetes is a major risk factor for cardiovascular disease and preventive measures, both lifestyle and pharmacological, are essential to prevent complications (18). To assess disease risk a combination of parameters is addressed, including blood pressure, blood lipids and glycaemic control, all of which are causally related to overweight and obesity (19-21). 
The aims of this study were to compare the strength and utility of WHtR with other anthropometric measures in terms of disease risk, and to consider the most appropriate cut point(s) for its use in patients with type 2 diabetes at high risk of cardiovascular disease, in a large international study with high-quality data: the Action in Diabetes and Vascular Disease: Preterax and Diamicron Modified Release Controlled Evaluation (ADVANCE) factorial trial and, in its post-trial follow-up, the ADVANCE-Observational Study (ADVANCE-ON).

\section{Methods}

Study design, characteristics of participants, and the main results of the ADVANCE trial and ADVANCE-ON study have previously been published $(22,23)$. In brief, participants from 20 countries, 55 years or older at high risk of a cardiovascular event were randomly assigned to an intensive glucose-control regimen, aiming for an $\mathrm{HbA} 1 \mathrm{c}-\mathrm{value} \mathrm{d} 6.5 \%$, or to standard glucose control, with targets based on local guidelines. Participants were also randomly assigned to a fixed-dose combination of perindopril $(4 \mathrm{mg})$ and indapamide $(1.25 \mathrm{mg})$ or matching placebo (24). 8494 surviving participants were enrolled in ADVANCE-ON (23). Overall, the median follow-up time in ADVANCE plus ADVANCE-ON was 9.0 years.

\footnotetext{
Anthropometric measurements

Besides WHtR, three other anthropometric measures were assessed as predictors of cardiovascular disease: body mass index (BMI), waist circumference (WC) and waist hip ratio (WHR). BMI was calculated as weight $(\mathrm{kg})$ divided by the square of height $(\mathrm{m})$, WHR was calculated as WC $(\mathrm{cm})$ divided by hip circumference $(\mathrm{cm})$ and WHtR was calculated as WC $(\mathrm{cm})$ divided by height $(\mathrm{cm})$.
} 


\section{Major outcomes}

The primary outcome was CVD, defined in ADVANCE as a major macrovascular event which is a composite endpoint of death from cardiovascular causes, nonfatal myocardial infarction or nonfatal stroke. Secondary outcomes were fatal or nonfatal myocardial infarction, fatal or nonfatal stroke, cardiovascular death and death from any cause.

\section{Statistical methods}

Categorical variables were summarized as the number of patients with corresponding percentage. Continuous variables were summarized as mean (SD) or, for those with a skewed distribution (25), median (interquartile range). WHtR values corresponding to BMI cut points defined by WHO $\left(18.5,25,30,35\right.$ and $\left.40 \mathrm{~kg} / \mathrm{m}^{2}\right)(26)$ were estimated using linear regression analyses. The same approach was used to estimate WHtR values corresponding to sex and region specific WC cut points (7) (Asian men - 90 and $102 \mathrm{~cm}$, non-Asian men - 94 and 102 $\mathrm{cm}$ and women -80 and $88 \mathrm{~cm}$ ). Participants classified as Asian were of Asian domicile. The linearity of associations of each anthropometric variable with each outcome, on the log scale, were tested by fitting, and comparing, ordinal and categorical Cox proportional hazard regression models (27). To describe the association of the different anthropometric measurements with the primary outcome, and any other outcome that showed evidence of non-linearity, restricted cubic splines were used in Cox regressions with pre-specified adjustment for age, sex, current cigarette smoking, region (Asian/not), randomised blood pressure-lowering intervention and randomised glucose control intervention. Further adjusted Cox models were then fitted for each anthropometric variable - outcome relationship that was approximately linear on the log scale, for a one standard deviation (across all subjects) increase, and for non-linear relationships after dividing the anthropometric variable into its 
constituent quarters. The proportional hazards assumption was explored through Schoenfeld residuals, showing results from associated tests. Bootstrapping (with 1000 repetitions) was then used to estimate the ratio of hazard ratios (and 95\% confidence intervals) comparing pairs of anthropometric variables in relation to the primary outcome (27). Finally, participants were categorised into three groups according to WHtR cut points derived from the regression analyses against BMI done earlier (corresponding WHtR values of BMI cut points for normal weight, overweight and obesity), and adjusted Cox models fitted for all outcomes. Sensitivity analyses with additional adjustment of age at completion of highest level of formal education as well as history of macrovascular disease and duration of diabetes were also carried out.

\section{Results}

Of the 11140 participants in ADVANCE, 15 who lacked baseline anthropometric measurements were excluded, so that data from 11125 (42\% female and 37\% Asian) participants were used in these analyses (Table 1).

Compared to women, men had more prior macrovascular disease than women at baseline, and were more frequently smokers. Women were more frequently treated with antihypertensive medications than men, but less frequently with statins. Men were heavier than women and had a greater median WHR as well as WC. Women, however, had a greater BMI and WHtR than men.

Asian participants were younger than non-Asians at the diagnosis of diabetes, had more commonly had a previous stroke and were less often treated with antihypertensives or statins 
than non-Asian participants. Asians weighed less than non-Asians, had a smaller WC, a lower BMI and a lower WHtR than non-Asians (Supplementary Table 1). Participants below and above median age were approximately the same weight, but the median WHtR was greater in participants younger than 66 years.

Figure 1 and Supplementary Table 2a shows the estimated equivalent WHtR cut points to the recommended BMI cut points according to region. These cut points were consistent across subpopulations with values of 0.47 and 0.48 for Asian and non-Asian domicile corresponding to BMI $18.5 \mathrm{~kg} / \mathrm{m}^{2} ; 0.55$ and 0.56 corresponding to a BMI of $25 \mathrm{~kg} / \mathrm{m}^{2}$; and 0.62 in both cases corresponding to a BMI of $30 \mathrm{~kg} / \mathrm{m}^{2}$. Thus cut-points for WHtR of approximately 0.55 and 0.6 would associate with the standard BMI cut-points of 25 and $30 \mathrm{~kg} / \mathrm{m}^{2}$. Observed WHtR values were less consistent when compared to standard WC cut offs, with lower corresponding WHtR cut points in Asian and Non-Asian women compared to Asian and Non-Asian men (Supplementary Figure 1; Supplementary Table 2b).

\section{Primary outcome - major macrovascular event}

During follow-up, 2162 (19\%) had a major macrovascular event. Figure 2 shows spline curves for the association of the primary outcome with all the anthropometric measurements tested, revealing approximately log-linear associations for all, but with a steeper slope for WHtR and WC. There was no evidence of non-linearity for any of the anthropometric variables (Supplementary Table 3). For the WHtR association there was a shallow slope between 0.5 and 0.55 but a steeper slope between 0.55 and 0.6 , suggesting that 0.55 might be a more appropriate cut-point for WHtR in these diabetes data than the value of 0.5 suggested in the literature. This is in agreement with the findings of Figure 1. There was some evidence 
of non-proportional hazards for BMI, which suggests that estimates for this variable should be viewed as averages over follow-up time (Supplementary Table 4; Supplementary Figure 2).

WHtR was equal to, and better than BMI and waist hip ratio across the entire range of values for these variables (Figure 3). The hazard ratio (HR) (confidence interval $[\mathrm{CI}]$ ) for a major macrovascular event for a one standard deviation (SD) higher WHtR was $1.16(1.11-1.22)$. For BMI, WHR and WC the corresponding HR (CI) was $1.09(1.04-1.14), 1.09$ (1.04$1.15)$ and $1.15(1.09-1.21)$ respectively. Additional adjustments for education, history of macrovascular disease and duration of diabetes did not change the results (Supplementary Table 5). When the anthropometric measurements were compared by bootstrapping, WHtR and WC were significantly better than BMI and WHR, but there was no significant difference between WHtR and WC (Supplementary Figure 3). There was no interaction by age, sex or region for the association between WHtR and the primary outcome (Supplementary Table 6).

Table 2 shows results for the primary outcome with participants divided into three categories depending on the WHtR cut points found from Figure 1. Relative to WHtR $<0.55$, the $\mathrm{HR}$ (CI) for a WHtR of $0.55-<0.6$ was 1.27 (CI 1.13-1.42) and for a WHtR of e 0.6, was 1.44 (CI 1.29-1.61); $\mathrm{p}$-value for trend $<0.0001$. There was no interaction between subgroups defined by sex and region, but there was a significantly greater effect of increasing WHtR in older, compared to younger, people. 


\section{Secondary outcomes}

During follow-up, 721 (7\%) participants had a myocardial infarction, 966 (9\%) had a stroke, 987 (9\%) died from cardiovascular causes and 2261 (20\%) died from any cause. For all these secondary outcomes, except all-cause mortality (Supplementary Table 3; Supplementary Figure 4; Supplementary Table 7), there was no evidence of non-linearity. There was no evidence of non-proportionality of hazards, except for WC related to MI (Supplementary Table 4; Supplementary Figure 2). WHtR was positively associated with all secondary outcomes (Figure 3; Supplementary Table 4). Additional adjustments for education, history of macrovascular disease and duration of diabetes did not change the results (Supplementary Table 5). Compared to a WHtR of $<0.55$, participants with a WHtR of e 0.6 , but not $0.55-$ $<0.6$, were at a significantly increased risk of myocardial infarction and stroke (Supplementary Table 8). The risk of cardiovascular death and all-cause death were significantly increased for both the other WHtR categories compared to the reference. WHtR was also associated with all secondary outcomes across subgroups, except stroke (Supplementary Table 6).

\section{Discussion}

This study showed that WHtR was as effective as waist circumference and better than BMI and waist to hip ratio for CVD risk prediction in our study population with diabetes. A WHtR of 0.48 to 0.74 corresponded to a BMI of 18.5 to $40 \mathrm{~m} / \mathrm{kg}^{2}$ and the association between WHtR and risk of a major macrovascular event was linear, with no suggestion of a J-shaped curve. WHtR was consistent across age, sex and region. We were not able to show clear evidence of the worth of the suggested cut point for WHtR of $0.5(12,13)$ for increased risk of cardiovascular disease in this cohort of patients with type 2 diabetes; indeed, our analyses 
suggest that a value of 0.55 might be more appropriate. There was an increased risk for a major cardiovascular event, cardiovascular death and death from any cause in those with a WHtR of e 0.55 compared to a WHtR $<0.55$, but not for myocardial infarction and stroke. A WHtR of e 0.6 was associated to increased cardiovascular disease risk for all subgroups except for stroke in participants aged 66 years or above.

WHtR has previously been found to align well across different age groups, genders and ethnicities $(13,28,29)$, which is in alignment with our findings. A meta-analysis of elderly persons showed that WHtR was a better predictor for cardiovascular risk in younger elderly persons compared to older elderly persons (15). In ADVANCE-ON, participants had a median age of 66 and the 90th percentile was 74 years, thus there were few older elderly participants, and we were therefore unable to assess the cardiovascular risk for elderly type 2 diabetes patients. In general, patients with type 2 diabetes, and hence all participants in ADVANCE-ON were at higher risk for a cardiovascular event compared to the general population.

To date, there is limited evidence of the utility of WHtR to assess the risk of CVD in patients with type 2 diabetes. In the general population, WHtR has been found to better predict CVD compared to $\mathrm{WC}(13,30)$ and $\mathrm{BMI}(13,31)$, where $\mathrm{BMI}$ has been repeatedly shown to preform worse than WHtR and WC $(13,31-33)$. A suitable WHtR cut point for increased cardiometabolic risk in the general population approximates 0.5 in several previous studies 
$(12,28,31,34)$. In a study of Han and Uygur Chinese people with diabetes, the suggested cut point for increased cardio-metabolic risk was 0.6 for men and women in both ethnic groups (35). In a subsample of Australian indigenous study participants with diabetes, an optimal cut point for cardiometabolic risk was suggested to be 0.6 as well (36). Both these studies in patients with diabetes align well with our findings.

WC is widely used and recommended for clinical practice (37). We could not show that WHtR was superior to WC in prediction of cardiovascular risk. WC is simpler than WHtR and requires only a tape measure and no calculations. However, for WC different suggested cut points have to be taken into account for different sexes, regions and ages, whereas pertinent cut points for WHtR appear to remain consistent across these subgroups for all outcomes tested here.

In terms of stroke, the association between WHtR and stroke in patients with type 2 diabetes needs further study and could possibly be related to other risk factors, such as blood pressure and could vary with different stroke subtypes (38).

The strengths of this study were the multi-ethnical study population, with patients with type 2 diabetes participating from 20 countries, the size of the study population, as well as the follow-up time of over 9 years. Limitations were that the sample consisted of a trial study population which may not be representative of the general population; the study was limited 
to patients with type 2 diabetes; and the ADVANCE protocol excluded patients aged less than 55 years whilst the most elderly patients, aged 80 years or more, were poorly represented in our study population. This paper takes no account of mediating factors in the causal pathway between anthropometry and outcomes, which would be an excellent topic for a future research.

\section{Conclusion}

Waist to height ratio is at least as effective as waist circumference, and preforms better than $\mathrm{BMI}$ and waist to hip ratio as a cardiovascular risk predictor in patients with type 2 diabetes at high risk of cardiovascular complications. Our findings are consistent across age, sex and region. WHtR is simple and easy to use, and has the advantage of the same suggested cut point across age, sex and region. For patients with type 2 diabetes the unofficial cut point of 0.5 , which has been suggested by some to be appropriate in general populations, may be too low to predict cardiovascular complications. There is some evidence to support a cut point of 0.55 or 0.6 but this needs further evaluation.

This article is protected by copyright. All rights reserved. 


\section{Author contributions}

K.R., J.C., and M.W. contributed to the concept and rationale for the study, interpretation of the results and drafted the manuscript. K.R. and T.O. conducted statistical analysis with advice from M.W. K.R., J.C., T.O., S.P., N.P., P.H., S.H. and M.W. contributed to discussion and reviewed and edited the manuscript. M.W. and J.C. are the guarantors of this work and, as such, had full access to all of the data in the study and take responsibility for the integrity of the data and the accuracy of the data analysis.

\section{Funding}

The ADVANCE trial was funded by grants from the National Health and Medical Research Council of Australia and Servier. K.R. is funded by a County Council of Östergötland international fellowship. T.O. is supported by a JSPS Overseas Research Fellowship. J.C. received research grants from the National Health and Medical Research Council of Australia and from Servier for the ADVANCE trial and ADVANCE-ON post-trial follow-up. S.P. is supported by a UK Medical Research Council Skills Development Fellowship (MR/P014550/1). M.W. is supported by a NHMRC Fellowship (APP108026).

\section{Conflict of interest statement}

Dr Chalmers, receiving research grants, lecture fees and travel support from Servier; Dr. Hamet, receiving consulting fees from Servier; Dr. Harrap, receiving lecture fees from Servier, Takeda, and Novartis; Dr Poulter received honoraria from Servier Laboratories, Takeda Pharmaceutical Company, Menarini Group, and Pfizer, grant support from Servier Laboratories, and Pfizer and personal fees from Servier Laboratories, Takeda Pharmaceutical 
Company, Menarini Group, and Pfizer; Dr Woodward reports consultancy fees from Amgen.

No other potential conflict of interest relevant to this article was reported.

\section{References}

1. Snijder MB, van Dam RM, Visser M, Seidell JC. What aspects of body fat are particularly hazardous and how do we measure them? Int J Epidemiol. 2006;35(1):83-92.

2. Larsson B, Svardsudd K, Welin L, Wilhelmsen L, Bjorntorp P, Tibblin G. Abdominal adipose tissue distribution, obesity, and risk of cardiovascular disease and death: 13 year follow up of participants in the study of men born in 1913. Br Med J (Clin Res Ed). 1984;288(6428):1401-4.

3. Asia Pacific Cohort Studies Collaboration, Central obesity and risk of cardiovascular disease in the Asia Pacific Region. Asia Pac J Clin Nutr. 2006;15(3):287-92.

4. World Health Organization (WHO) fact sheet Obesity and overweight http://www.who.int/mediacentre/factsheets/fs311/en/ accessed November 9th 2017. 2017.

5. World Health Organization (WHO). Waist Circumference and Waist-to Hip Ratio: Report of a WHO Expert Consultation, Geneva, 8-11 December. Geneva, WHO, 2008; . 2011.

6. Maynard LM, Wisemandle W, Roche AF, Chumlea WC, Guo SS, Siervogel RM. Childhood body composition in relation to body mass index. Pediatrics. 2001;107(2):344-50.

7. World Health Organization (WHO). Waist circumference and waist-hip ratio. Report of a WHO Expetrt Consultation. . 2008.

8. WHO Expert Consultation. Appropriate body-mass index for Asian populations and its implications for policy and intervention strategies. Lancet. 2004;363(9403):157-63.

9. Katzmarzyk PT, Bray GA, Greenway FL, Johnson WD, Newton RL, Jr., Ravussin E, et al. Ethnic-specific BMI and waist circumference thresholds. Obesity (Silver Spring). 2011;19(6):1272-8.

10. Ashwell M, Cole TJ, Dixon AK. Ratio of waist circumference to height is strong predictor of intra-abdominal fat. BMJ. 1996;313(7056):559-60.

11. Browning LM, Hsieh SD, Ashwell M. A systematic review of waist-to-height ratio as a screening tool for the prediction of cardiovascular disease and diabetes: 0.5 could be a suitable global boundary value. Nutr Res Rev. 2010;23(2):247-69. 
12. Ashwell M, Gibson S. A proposal for a primary screening tool: 'Keep your waist circumference to less than half your height'. BMC Med. 2014;12:207.

13. Ashwell M, Gunn P, Gibson S. Waist-to-height ratio is a better screening tool than waist circumference and BMI for adult cardiometabolic risk factors: systematic review and meta-analysis. Obes Rev. 2012;13(3):275-86.

14. Pan J, Wang M, Ye Z, Yu M, Shen Y, He Q, et al. Optimal cut-off levels of obesity indices by different definitions of metabolic syndrome in a southeast rural Chinese population. J Diabetes Investig. 2016;7(4):594-600.

15. Correa MM, Thume E, De Oliveira ER, Tomasi E. Performance of the waist-to-height ratio in identifying obesity and predicting non-communicable diseases in the elderly population: A systematic literature review. Arch Gerontol Geriatr. 2016;65:174-82.

16. Burns RD, Brusseau TA, Fang Y, Fu Y, Hannon JC. Establishing Waist-to-Height Ratio Standards from Criterion-Referenced BMI Using ROC Curves in Low-Income Children. J Obes. 2016;2016:2740538.

17. Ashwell M, Lejeune S, McPherson K. Ratio of waist circumference to height may be better indicator of need for weight management. BMJ. 1996;312(7027):377.

18. Fox CS, Golden SH, Anderson C, Bray GA, Burke LE, de Boer IH, et al. Update on Prevention of Cardiovascular Disease in Adults With Type 2 Diabetes Mellitus in Light of Recent Evidence: A Scientific Statement From the American Heart Association and the American Diabetes Association. Diabetes Care. 2015;38(9):1777-803.

19. Look ARG, Wing RR. Long-term effects of a lifestyle intervention on weight and cardiovascular risk factors in individuals with type 2 diabetes mellitus: four-year results of the Look AHEAD trial. Arch Intern Med. 2010;170(17):1566-75.

20. American Diabetes Association. Standards of Medical Care in Diabetes-2017. Diabetes Care. 2017;40(Suppl. 1):S1-S135.

21. Lu Y, Hajifathalian K, Ezzati M, Woodward M, Rimm EB, Danaei G. Metabolic mediators of the effects of body-mass index, overweight, and obesity on coronary heart disease and stroke: a pooled analysis of 97 prospective cohorts with 1.8 million participants. Lancet. 2014;383(9921):970-83.

22. Patel A, MacMahon S, Chalmers J, Neal B, Billot L, Woodward M, et al. Intensive blood glucose control and vascular outcomes in patients with type 2 diabetes. N Engl J Med. 2008;358(24):2560-72.

23. Zoungas S, Chalmers J, Neal B, Billot L, Li Q, Hirakawa Y, et al. Follow-up of blood-pressure lowering and glucose control in type 2 diabetes. N Engl J Med.

2014;371(15):1392-406. 
24. Rationale and design of the ADVANCE study: a randomised trial of blood pressure lowering and intensive glucose control in high-risk individuals with type 2 diabetes mellitus. Action in Diabetes and Vascular Disease: PreterAx and DiamicroN Modified-Release Controlled Evaluation. J Hypertens Suppl. 2001;19(4):S21-8.

25. Zhang J, Wu, Y. Likelihood-ratio tests for normality. Computational Statistics \& Data Analysis. 2004;49(2005):709-21.

26. WHO health topics, disease prevention, body mass index, BMI. http://www.euro.who.int/en/health-topics/disease-prevention/nutrition/a-healthylifestyle/body-mass-index-bmi.

27. Woodward M. Epidemiology, Study Design and Data Analysis, 3rd edition. 2014.

28. Ware LJ, Rennie KL, Kruger HS, Kruger IM, Greeff M, Fourie CM, et al. Evaluation of waist-to-height ratio to predict 5 year cardiometabolic risk in sub-Saharan African adults. Nutr Metab Cardiovasc Dis. 2014;24(8):900-7.

29. Peng Y, Li W, Wang Y, Bo J, Chen H. The Cut-Off Point and Boundary Values of Waist-to-Height Ratio as an Indicator for Cardiovascular Risk Factors in Chinese Adults from the PURE Study. PLoS One. 2015;10(12):e0144539.

30. Tatsumi Y, Watanabe M, Kokubo Y, Nishimura K, Higashiyama A, Okamura T, et al. Effect of age on the association between waist-to-height ratio and incidence of cardiovascular disease: the Suita study. J Epidemiol. 2013;23(5):351-9.

31. Savva SC, Lamnisos D, Kafatos AG. Predicting cardiometabolic risk: waist-to-height ratio or BMI. A meta-analysis. Diabetes Metab Syndr Obes. 2013;6:403-19.

32. Petursson H, Sigurdsson JA, Bengtsson C, Nilsen TI, Getz L. Body configuration as a predictor of mortality: comparison of five anthropometric measures in a 12 year follow-up of the Norwegian HUNT 2 study. PLoS One. 2011;6(10):e26621.

33. Schneider HJ, Glaesmer H, Klotsche J, Bohler S, Lehnert H, Zeiher AM, et al. Accuracy of anthropometric indicators of obesity to predict cardiovascular risk. J Clin Endocrinol Metab. 2007;92(2):589-94.

34. Yang H, Xin Z, Feng JP, Yang JK. Waist-to-height ratio is better than body mass index and waist circumference as a screening criterion for metabolic syndrome in Han Chinese adults. Medicine (Baltimore). 2017;96(39):e8192.

35. He CH, Pan S, Ma YT, Yang YN, Ma X, Li XM, et al. Optimal waist-to-height ratio cutoff values for predicting cardio-metabolic risk in Han and Uygur adults in northwest part of China. Eur J Clin Nutr. 2015;69(8):954-60. 
36. Li M, McDermott RA. Using anthropometric indices to predict cardio-metabolic risk factors in Australian indigenous populations. Diabetes Res Clin Pract. 2010;87(3):401-6.

37. Klein S, Allison DB, Heymsfield SB, Kelley DE, Leibel RL, Nonas C, et al. Waist circumference and cardiometabolic risk: a consensus statement from shaping America's health: Association for Weight Management and Obesity Prevention; NAASO, the Obesity Society; the American Society for Nutrition; and the American Diabetes Association. Diabetes Care. 2007;30(6):1647-52.

38. Hyun KK, Huxley RR, Arima H, Woo J, Lam TH, Ueshima H, et al. A comparative analysis of risk factors and stroke risk for Asian and non-Asian men: the Asia Pacific cohort studies collaboration. Int J Stroke. 2013;8(8):606-11. 


\section{Figure legends}

Figure 1. Scatter plot and fitted regression line relating waist to height ratio to body mass index in ADVANCE, by region.

Established BMI cut points are 18.5 - normal weight, 25 - overweight, 30 - obesity class 1 , 35 - obesity class 2 and 40 - obesity class 3. Corresponding waist to height ratio are: 0.47 , $0.55,0.62,0.68$ and 0.74 (Asia) and; 0.48, 0.56, 0.62, 0.67 and 0.73 (Non-Asia), respectively.

Figure 2. Hazard ratios for the association between anthropometric measurements (body mass index, waist hip ratio, waist circumference and waist to height ratio) and major macrovascular event according to obesity indices.

The circles represent the points at which knots were placed (5, 25, 50, 75, 95 percentiles). Shaded areas represent the $95 \%$ confidence intervals. Models were adjusted for age, sex, current smoking habit, Asian region, randomised blood pressure-lowering intervention and randomised glucose control intervention. Values were trimmed at less than 1st percentile and greater than 99th percentile of each obesity index.

Figure 3. Hazard ratios and $95 \%$ confidence intervals per one standard deviation higher body mass index, waist hip ratio, waist circumference and waist to height ratio, respectively.

Models adjusted for age, sex, current smoking habit, Asian region, randomised blood pressure-lowering intervention, and randomised glucose control intervention.

Myocardial infarction: fatal or nonfatal myocardial infarction. Stroke: fatal or nonfatal stroke.

Note that all-cause mortality is omitted from this figure beacause its relationship is nonlinear. Supplementary Table 7 shows results for all-cause mortality by quarters of each anthropometric variable.

This article is protected by copyright. All rights reserved. 
Figure 1.
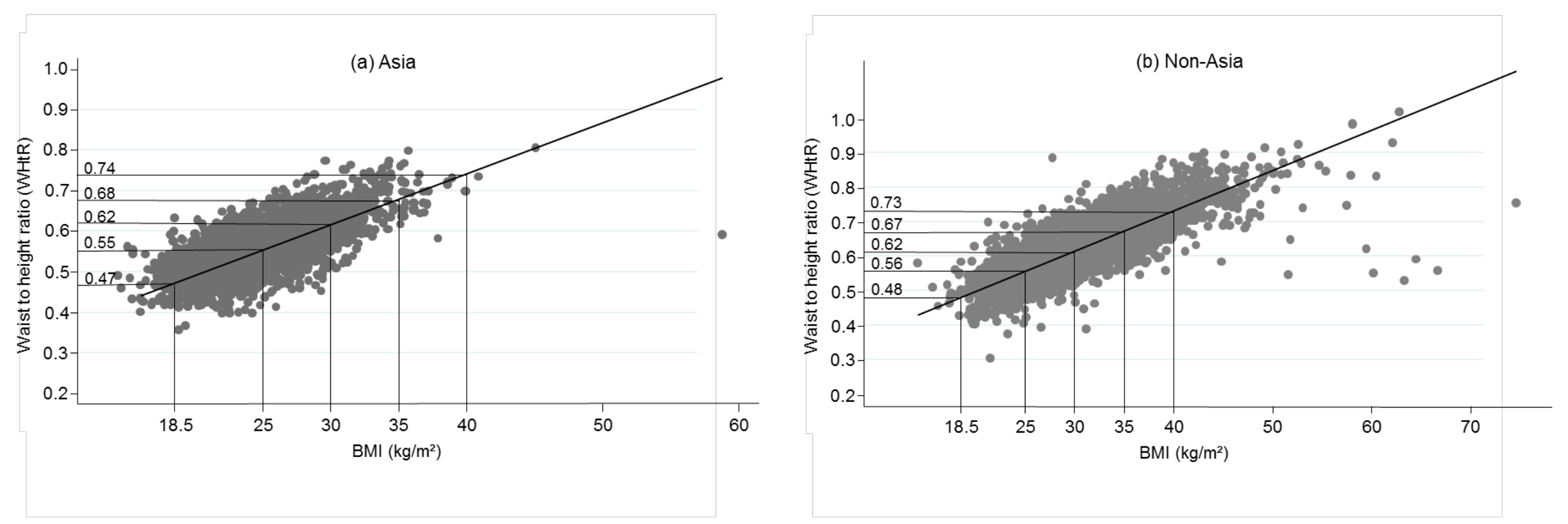

This article is protected by copyright. All rights reserved. 
Figure 2.
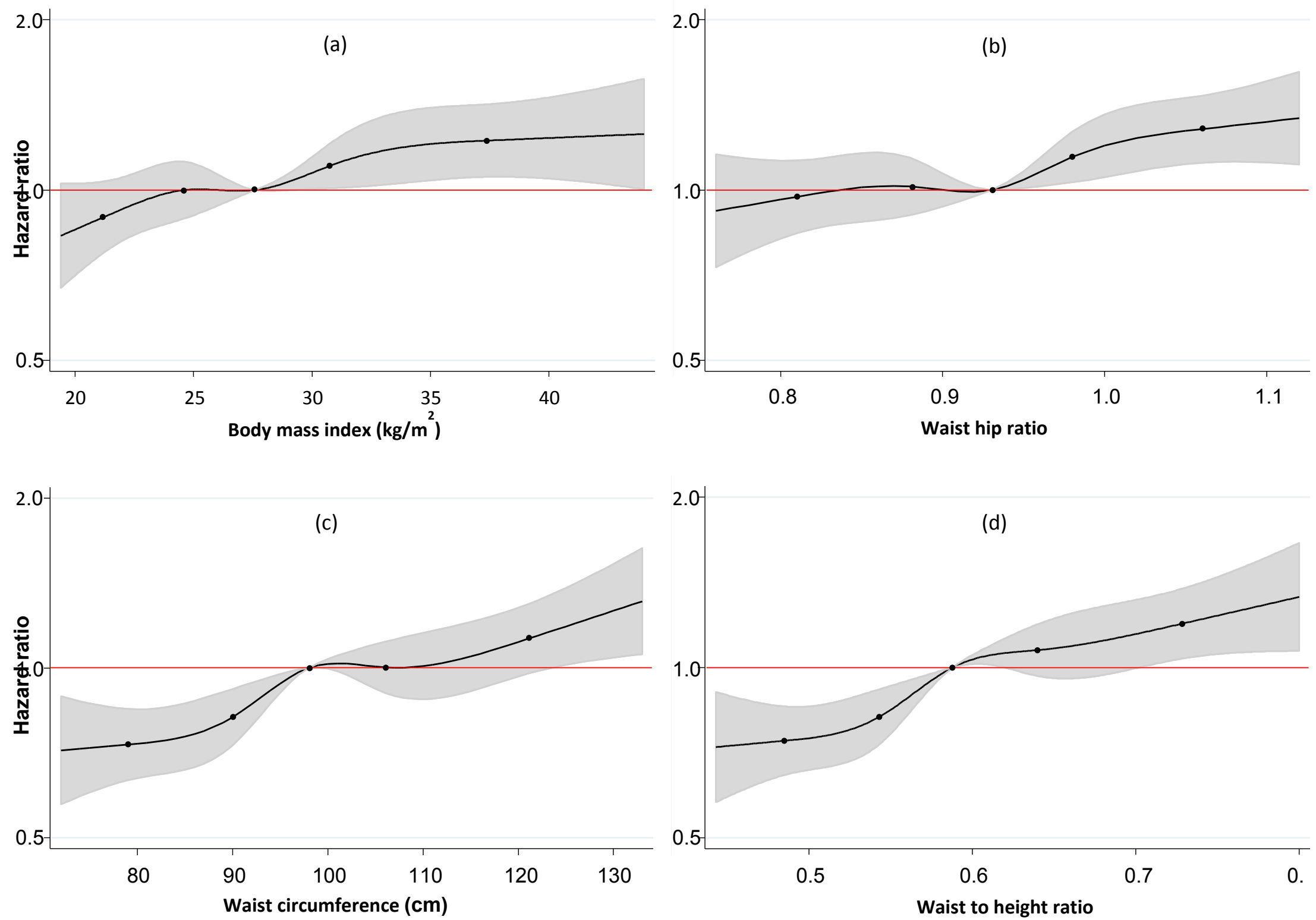

This article is protected by copyright. All rights reserved. 
Figure 3. Hazard ratios and $95 \%$ confidence intervals for primary and secondary outcomes per one standard deviation higher body mass index, waist hip ratio, waist circumference and waist to height ratio, respectively.

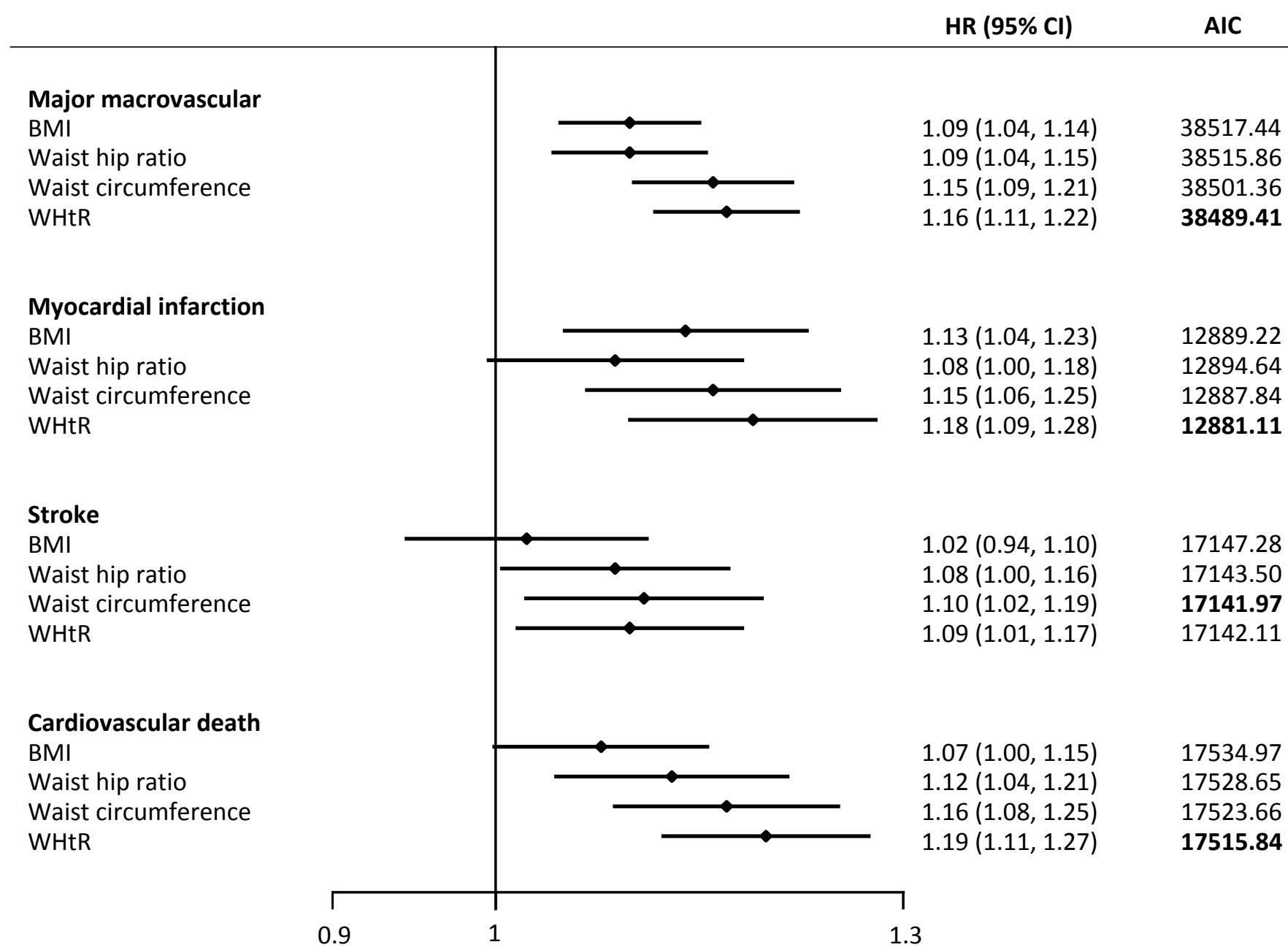

This article is protected by copyright. All rights reserved. 
Table 1. Baseline characteristics for 11125 patients with anthropometric measures from the ADVANCE trial: overall and according to sex.

\begin{tabular}{llll}
\hline & $\begin{array}{l}\text { Total } \\
\mathbf{n}=\mathbf{1 1 1 2 5}\end{array}$ & $\begin{array}{l}\text { Men } \\
\mathbf{n = 6 3 9 7}\end{array}$ & $\begin{array}{l}\text { Women } \\
\mathbf{n}=\mathbf{4 7 2 8}\end{array}$ \\
\hline Age, yr mean (SD) & $66(6)$ & $66(6)$ & $66(6)$ \\
\hline Age when diabetes first diagnosed, yr - mean (SD) & $58(9)$ & $58(9)$ & $58(9)$ \\
\hline Duration of diabetes, yr - mean (SD) & $7.9(6.4)$ & $8.0(6.5)$ & $7.9(6.2)$ \\
\hline Age at completion of highest education, yr - median & $18(7)$ & $19(7)$ & $17(7)$
\end{tabular}

\section{Anthropometric measures}

weight - median (Q1;03)

WC, cm - Median (Q1;Q3)

$\mathrm{BMI}$ - median (Q1;Q3)

WHR - median (Q1;Q3)

WHtR - median (Q1;Q3)

\section{Previous vascular disease}

History of major macrovascular disease - no. /total no.

(\%)

Myocardial infarction - no. /total no. (\%)

Stroke - no. /total no. (\%)

\begin{tabular}{l}
$76(66 ; 88)$ \\
$98(90 ; 106)$ \\
$28(25 ; 31)$ \\
$0.9(0.9 ; 1.0)$ \\
\hline $0.59(0.54 ; 0.64)$
\end{tabular}

$81(71 ; 92)$

$100(92 ; 108)$

$70(61 ; 81)$

$95(86 ; 103)$

$27(25 ; 30) \quad 28(25 ; 32)$

$1.0(0.9 ; 1.0) \quad 0.9(0.8 ; 0.9)$

$0.60(0.55 ; 0.65)$

Blood-glucose control

HbA1c level, \% - median (Q1;Q3)

Fasting blood glucose, $\mathrm{mmol} /$ liter - median (Q1;Q3)

$3583 / 11125(32)$

$2369 / 6397$ (37)

$1214 / 4728(26)$

\section{Other major risk factors}

Systolic blood pressure - mean (SD)

$1329 / 11125(12)$

$1005 / 6397(16) \quad 324 / 4728(7)$

$1021 / 11125$ (9)

$617 / 6397(10) \quad 404 / 4728(9)$

$7.2(6.5 ; 8.2)$

$7.2(6.5 ; 8.1)$

$7.3(6.5 ; 8.4)$

$7.9(6.6 ; 9.7)$

$7.9(6.6 ; 9.6)$

$8.0(6.6 ; 9.9)$

$145(22)$

$145(21)$

$145(22)$

This article is protected by copyright. All rights reserved. 


\begin{tabular}{llll}
\hline Diastolic blood pressure - mean (SD) & $81(11)$ & $81(11)$ & $80(11)$ \\
\hline Serum cholesterol, mmol/liter - median (Q1;Q3) & $5.1(4.4 ; 5.9)$ & $4.8(4.2 ; 5.6)$ & $5.5(4.7 ; 6.3)$ \\
\hline Low-density lipoprotein - median (Q1;Q3) & $3.0(2.4 ; 3.7)$ & $2.8(2.3 ; 3.5)$ & $3.2(2.6 ; 4.0)$ \\
\hline High-density lipoprotein - median (Q1;Q3) & $1.2(1.0 ; 1.4)$ & $1.1(1.0 ; 1.4)$ & $1.3(1.1 ; 1.5)$ \\
\hline Current smoking - no./total no. (\%) & $1549 / 11125(14)$ & $1115 / 6397(17)$ & $434 / 4728(9)$ \\
\hline
\end{tabular}

\section{Treatment at baseline}

\begin{tabular}{llll}
\hline On antihypertensive treatment - no./ total no. (\%) & $7645 / 11125(69)$ & $4198 / 6397(66)$ & $3447 / 4728(73)$ \\
\hline Statin - no./ total no. (\%) & $3142 / 11125(28)$ & $2011 / 6397(31)$ & $1131 / 4728(24)$ \\
\hline Other lipid-modifying drug - no./ total no. (\%) & $933 / 11125(8)$ & $513 / 6397(8)$ & $420 / 4728(9)$ \\
\hline Aspirin - no./ total no. (\%) & $4889 / 11125(44)$ & $3056 / 6397(48)$ & $1833 / 4728(39)$ \\
\hline Other antiplatelet agent - no./ total no. (\%) & $504 / 11125(5)$ & $328 / 6397(5)$ & $176 / 4728(4)$ \\
\hline Oral anticoagulants - no./ total no. (\%) & $408 / 11125(4)$ & $255 / 6397(4)$ & $153 / 4728(3)$ \\
\hline Number of glucose lowering agents - median (Q1:Q3) & $1.0(1.0 ; 2.0)$ & $1.0(1.0 ; 2.0)$ & $1.0(1.0 ; 2.0)$ \\
\hline Gliclazide (modified release) - no./ total no. (\%) & $865 / 11125(8)$ & $468 / 6397(7)$ & $397 / 4728(8)$ \\
\hline Other sulfonurea - no./ total no. (\%) & $7078 / 11124(64)$ & $4138 / 6397(65)$ & $2940 / 4727(62)$ \\
\hline Metformin - no./ total no. (\%) & $6747 / 11125(61)$ & $3847 / 6397(60)$ & $2900 / 4728(61)$ \\
\hline Thiazolidinedione - no./ total no. (\%) & $405 / 11125(4)$ & $265 / 6397(4)$ & $140 / 4728(3)$ \\
\hline Insulin - no./ total no. (\%) & $159 / 11125(1)$ & $66 / 6397(1)$ & $93 / 4728(2)$
\end{tabular}

Data are $\mathrm{n}(\%)$ for categorical variables, mean (SD) for normally distributed continuous variables or median (Q1:Q3) for non-normally distributed continuous variables. Distribution was checked by use of the Anderson-Darling test.

WHR - waist hip ratio, BMI - body mass index, WC - waist circumference, WHtR - waist to height ratio

This article is protected by copyright. All rights reserved. 
Table 2. Hazard ratios (95\% confidence Interval) for major macrovascular event according to three waist to height ratio (WHtR) categories.

WHtR $<0.55$ WHtR $0.55-<0.6$ WHtR $>=0.6 \quad p$ for interaction

\begin{tabular}{lllll}
\hline Participants $-\mathbf{n}(\%)$ & & & & \\
Men & $1945(30)$ & $1904(30)$ & $2548(40)$ & \\
Women & $1225(26)$ & $1178(25)$ & $2325(49)$ & \\
Asian & $1967(47.6)$ & $1291(31.2)$ & $874(21.2)$ & \\
Non-Asian & $1203(17.2)$ & $1791(25.6)$ & $3999(57.2)$ & \\
Age $<66$ years & $1620(30.6)$ & $1413(26.7)$ & $2258(42.7)$ & \\
Age $>=66$ years & $1550(26.6)$ & $1669(28.6)$ & $2615(44.8)$ & \\
All & $3170(28)$ & $3082(28)$ & $4873(44)$ & \\
Major macrovascular event & & & & \\
Men & 1 (reference) & $1.22(1.06-1.40)$ & $1.39(1.20-1.60)$ & 0.7668 \\
Women & 1 (reference) & $1.35(1.11-1.65)$ & $1.53(1.27-1.84)$ & \\
Asian & 1 (reference) & $1.24(1.07-1.44)$ & $1.48(1.25-1.74)$ & 0.8045 \\
Non-Asian & 1 (reference) & $1.30(1.08-1.56)$ & $1.45(1.23-1.72)$ & \\
Age $<66$ years & 1 (reference) & $1.09(0.92-1.31)$ & $1.18(1.01-1.39)$ & $<0.0001$ \\
Age $>=66$ years & 1 (reference) & $1.25(1.08-1.45)$ & $1.27(1.10-1.45)$ & \\
All & 1 (reference) & $1.27(1.13-1.42)$ & $1.44(1.29-1.61)$ & \\
\hline
\end{tabular}

Adjusted for age, sex, current cigarette smoking, region, randomised blood pressure-lowering intervention and randomised glucose control intervention. P for trend was significant for all participants as well as sex, region and age subgroups. P for interaction between subgroups and WHtR categories are shown.

This article is protected by copyright. All rights reserved. 
Table 1. Baseline characteristics for 11125 patients with anthropometric measures from the ADVANCE trial: overall and according to sex.

\begin{tabular}{llll}
\hline & $\begin{array}{l}\text { Total } \\
\mathbf{n}=\mathbf{1 1 1 2 5}\end{array}$ & $\begin{array}{l}\text { Men } \\
\mathbf{n = 6 3 9 7}\end{array}$ & $\begin{array}{l}\text { Women } \\
\mathbf{n}=\mathbf{4 7 2 8}\end{array}$ \\
\hline Age, yr mean (SD) & $66(6)$ & $66(6)$ & $66(6)$ \\
\hline Age when diabetes first diagnosed, yr - mean (SD) & $58(9)$ & $58(9)$ & $58(9)$ \\
\hline Duration of diabetes, yr - mean (SD) & $7.9(6.4)$ & $8.0(6.5)$ & $7.9(6.2)$ \\
\hline Age at completion of highest education, yr - median & $18(7)$ & $19(7)$ & $17(7)$
\end{tabular}

(Q1;Q3)

\section{Anthropometric measures}

\begin{tabular}{llll}
\hline weight - median (Q1;Q3) & $76(66 ; 88)$ & $81(71 ; 92)$ & $70(61 ; 81)$ \\
\hline WC, cm - Median (Q1;Q3) & $98(90 ; 106)$ & $100(92 ; 108)$ & $95(86 ; 103)$ \\
\hline BMI, - median (Q1;Q3) & $28(25 ; 31)$ & $27(25 ; 30)$ & $28(25 ; 32)$ \\
\hline WHR - median (Q1;Q3) & $0.9(0.9 ; 1.0)$ & $1.0(0.9 ; 1.0)$ & $0.9(0.8 ; 0.9)$ \\
\hline WHtR - median (Q1;Q3) & $0.59(0.54 ; 0.64)$ & $0.58(0.54 ; 0.63)$ & $0.60(0.55 ; 0.65)$
\end{tabular}

\section{Previous vascular disease}

History of major macrovascular disease - no. /total no. $\quad 3583 / 11125$ (32) $\quad 2369 / 6397$ (37) $\quad 1214 / 4728$ (26)

(\%)

Myocardial infarction - no. /total no. (\%)

$1329 / 11125(12) \quad 1005 / 6397(16) \quad 324 / 4728(7)$

Stroke - no. /total no. (\%)

$1021 / 11125(9) \quad 617 / 6397(10) \quad 404 / 4728(9)$

\section{Blood-glucose control}

HbA1c level, \% - median (Q1;Q3)

$7.2(6.5 ; 8.2)$

$7.2(6.5 ; 8.1)$

$7.3(6.5 ; 8.4)$

Fasting blood glucose, mmol/liter - median (Q1;Q3)

$7.9(6.6 ; 9.7)$

$7.9(6.6 ; 9.6)$

$8.0(6.6 ; 9.9)$

Other major risk factors

Systolic blood pressure - mean (SD)

$145(22)$

$145(21)$

$145(22)$

This article is protected by copyright. All rights reserved. 


\begin{tabular}{llll}
\hline Diastolic blood pressure - mean (SD) & $81(11)$ & $81(11)$ & $80(11)$ \\
\hline Serum cholesterol, mmol/liter - median (Q1;Q3) & $5.1(4.4 ; 5.9)$ & $4.8(4.2 ; 5.6)$ & $5.5(4.7 ; 6.3)$ \\
\hline Low-density lipoprotein - median (Q1;Q3) & $3.0(2.4 ; 3.7)$ & $2.8(2.3 ; 3.5)$ & $3.2(2.6 ; 4.0)$ \\
\hline High-density lipoprotein - median (Q1;Q3) & $1.2(1.0 ; 1.4)$ & $1.1(1.0 ; 1.4)$ & $1.3(1.1 ; 1.5)$ \\
\hline Current smoking - no./total no. (\%) & $1549 / 11125(14)$ & $1115 / 6397(17)$ & $434 / 4728(9)$ \\
\hline
\end{tabular}

\section{Treatment at baseline}

\begin{tabular}{llll}
\hline On antihypertensive treatment - no./ total no. (\%) & $7645 / 11125(69)$ & $4198 / 6397(66)$ & $3447 / 4728(73)$ \\
\hline Statin - no./ total no. (\%) & $3142 / 11125(28)$ & $2011 / 6397(31)$ & $1131 / 4728(24)$ \\
\hline Other lipid-modifying drug - no./ total no. (\%) & $933 / 11125(8)$ & $513 / 6397(8)$ & $420 / 4728(9)$ \\
\hline Aspirin - no./ total no. (\%) & $4889 / 11125(44)$ & $3056 / 6397(48)$ & $1833 / 4728(39)$ \\
\hline Other antiplatelet agent - no./ total no. (\%) & $504 / 11125(5)$ & $328 / 6397(5)$ & $176 / 4728(4)$ \\
\hline Oral anticoagulants - no./ total no. (\%) & $408 / 11125(4)$ & $255 / 6397(4)$ & $153 / 4728(3)$ \\
\hline Number of glucose lowering agents - median (Q1:Q3) & $1.0(1.0 ; 2.0)$ & $1.0(1.0 ; 2.0)$ & $1.0(1.0 ; 2.0)$ \\
\hline Gliclazide (modified release) - no./ total no. (\%) & $865 / 11125(8)$ & $468 / 6397(7)$ & $397 / 4728(8)$ \\
\hline Other sulfonurea - no./ total no. (\%) & $7078 / 11124(64)$ & $4138 / 6397(65)$ & $2940 / 4727(62)$ \\
\hline Metformin - no./ total no. (\%) & $6747 / 11125(61)$ & $3847 / 6397(60)$ & $2900 / 4728(61)$ \\
\hline Thiazolidinedione - no./ total no. (\%) & $405 / 11125(4)$ & $265 / 6397(4)$ & $140 / 4728(3)$ \\
\hline Insulin - no./ total no. (\%) & $159 / 11125(1)$ & $66 / 6397(1)$ & $93 / 4728(2)$
\end{tabular}

Data are $\mathrm{n}(\%)$ for categorical variables, mean (SD) for normally distributed continuous variables or median (Q1:Q3) for non-normally distributed continuous variables. Distribution was checked by use of the Anderson-Darling test.

WHR - waist hip ratio, BMI - body mass index, WC - waist circumference, WHtR - waist to height ratio 
Table 2. Hazard ratios (95\% confidence Interval) for major macrovascular event according to three waist to height ratio (WHtR) categories.

WHtR $<0.55$ WHtR $0.55-<0.6$ WHtR $>=0.6 \quad p$ for interaction

\begin{tabular}{lllll}
\hline Participants $-\mathbf{n}(\%)$ & & & & \\
$\quad$ Men & $1945(30)$ & $1904(30)$ & $2548(40)$ & \\
Women & $1225(26)$ & $1178(25)$ & $2325(49)$ & \\
Asian & $1967(47.6)$ & $1291(31.2)$ & $874(21.2)$ & \\
Non-Asian & $1203(17.2)$ & $1791(25.6)$ & $3999(57.2)$ & \\
Age $<66$ years & $1620(30.6)$ & $1413(26.7)$ & $2258(42.7)$ & \\
Age $>=66$ years & $1550(26.6)$ & $1669(28.6)$ & $2615(44.8)$ & \\
All & $3170(28)$ & $3082(28)$ & $4873(44)$ & \\
Major macrovascular event & & & & \\
Men & 1 (reference) & $1.22(1.06-1.40)$ & $1.39(1.20-1.60)$ & 0.7668 \\
Women & 1 (reference) & $1.35(1.11-1.65)$ & $1.53(1.27-1.84)$ & \\
Asian & 1 (reference) & $1.24(1.07-1.44)$ & $1.48(1.25-1.74)$ & 0.8045 \\
Non-Asian & 1 (reference) & $1.30(1.08-1.56)$ & $1.45(1.23-1.72)$ & \\
Age $<66$ years & 1 (reference) & $1.09(0.92-1.31)$ & $1.18(1.01-1.39)$ & $<0.0001$ \\
Age $>=66$ years & 1 (reference) & $1.25(1.08-1.45)$ & $1.27(1.10-1.45)$ & \\
All & 1 (reference) & $1.27(1.13-1.42)$ & $1.44(1.29-1.61)$ & \\
\hline
\end{tabular}

Adjusted for age, sex, current cigarette smoking, region, randomised blood pressure-lowering intervention and randomised glucose control intervention. P for trend was significant for all participants as well as sex, region and age subgroups. P for interaction between subgroups and WHtR categories are shown. 


\section{University Library}

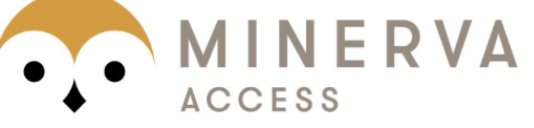

A gateway to Melbourne's research publications

Minerva Access is the Institutional Repository of The University of Melbourne

Author/s:

Radholm, K;Chalmers, J;Ohkuma, T;Peters, S;Poulter, N;Hamet, P;Harrap, S;Woodward, M

Title:

Use of the waist-to-height ratio to predict cardiovascular risk in patients with diabetes:

Results from the ADVANCE-ON study

Date:

2018-08-01

Citation:

Radholm, K., Chalmers, J., Ohkuma, T., Peters, S., Poulter, N., Hamet, P., Harrap, S. \& Woodward, M. (2018). Use of the waist-to-height ratio to predict cardiovascular risk in patients with diabetes: Results from the ADVANCE-ON study. DIABETES OBESITY \& METABOLISM, 20 (8), pp.1903-1910. https://doi.org/10.1111/dom.13311.

Persistent Link:

http://hdl.handle.net/11343/283783 